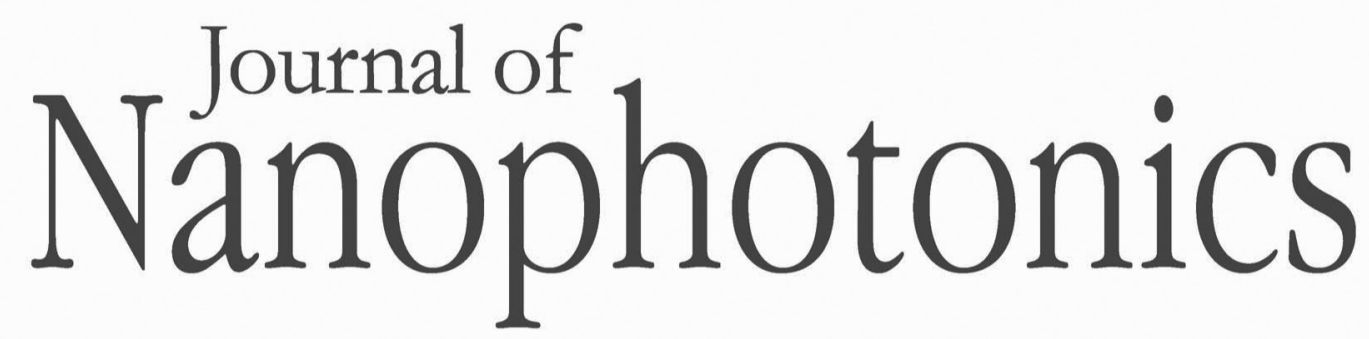

\title{
Integrated waveguide and nanostructured sensor platform for surface-enhanced Raman spectroscopy
}

Stuart J. Pearce

Michael E. Pollard

SweZin Oo

Ruiqi Chen

Sumit Kalsi

Martin D. B. Charlton

\section{O SPIE}




\title{
Integrated waveguide and nanostructured sensor platform for surface-enhanced Raman spectroscopy
}

\author{
Stuart J. Pearce,* Michael E. Pollard, SweZin Oo, Ruiqi Chen, \\ Sumit Kalsi, and Martin D. B. Charlton \\ University of Southampton, Electronics and Computer Science, Southampton, SO171BJ, \\ United Kingdom
}

\begin{abstract}
Limitations of current sensors include large dimensions, sometimes limited sensitivity and inherent single-parameter measurement capability. Surface-enhanced Raman spectroscopy can be utilized for environment and pharmaceutical applications with the intensity of the Raman scattering enhanced by a factor of $10^{6}$. By fabricating and characterizing an integrated optical waveguide beneath a nanostructured precious metal coated surface a new surface-enhanced Raman spectroscopy sensing arrangement can be achieved. Nanostructured sensors can provide both multiparameter and high-resolution sensing. Using the slab waveguide core to interrogate the nanostructures at the base allows for the emission to reach discrete sensing areas effectively and should provide ideal parameters for maximum Raman interactions. Thin slab waveguide films of silicon oxynitride were etched and gold coated to create localized nanostructured sensing areas of various pitch, diameter, and shape. These were interrogated using a Ti:Sapphire laser tuned to $785-\mathrm{nm}$ end coupled into the slab waveguide. The nanostructured sensors vertically projected a Raman signal, which was used to actively detect a thin layer of benzyl mercaptan attached to the sensors. (c) 2014 Society of Photo-Optical Instrumentation Engineers (SPIE) [DOI: 10 .1117/1.JNP.8.083989]
\end{abstract}

Keywords: surface-enhanced Raman spectroscopy; nanostructures; plasmons; finite-difference time-domain; Raman; waveguide.

Paper 13118SSP received Oct. 29, 2013; revised manuscript received Mar. 19, 2014; accepted for publication Mar. 20, 2014; published online Apr. 11, 2014.

\section{Introduction}

Raman spectroscopy is a useful analytical technique for the identification of molecules. A laser source interacts with an unknown chemical sample and the light scattered from the surface is detected and analyzed. Most of the incident wavelength will undergo elastic scattering, however, a small amount of the scattered light will undergo inelastic scattering over a range of different wavelengths. These wavelengths are indicative of the vibrational transitions in the molecules of the unknown chemical sample. These vibrational transitions are dependent on the molecules; therefore, the inelastic scattering can be used to identify functional subgroups of the test molecule. ${ }^{1}$ Devices capable of molecular detection are of great interest for many applications including cancer cell detection, ${ }^{2-4}$ drug analysis, ${ }^{5,6}$ and explosives detection. ${ }^{7,8}$

Although extremely useful for bulk sample identification, conventional Raman spectroscopy has limited applications as the level of detection of the inelastic-scattered light is very low. ${ }^{3}$ Several methods have been proposed to increase Raman scattering efficiency, including stimulated Raman processes and other resonance enhancements; however, the most significant amplification of the Raman signal is provided by surface-enhanced Raman spectroscopy (SERS).

SERS is a Raman spectroscopic technique that provides greatly enhanced Raman signal from Raman-active analyte molecules that have been adsorbed onto certain specially prepared metal surfaces. Since the first observations in $1974,{ }^{9}$ interest and applications for SERS have grown exponentially. In contrast to standard Raman spectroscopy SERS benefits from high sensitivity

*Address all correspondence to: Stuart J. Pearce, E-mail: sp3@ecs.soton.ac.uk

0091-3286/2014/\$25.00 (C) 2014 SPIE 
Pearce et al.: Integrated waveguide and nanostructured sensor platform for surface-enhanced...

and surface selectivity. ${ }^{10,11}$ This technique can be used to identify and quantify molecules, viruses, and bacteria in low quantities.

The benefit of SERS lies with its ability to effectively "fingerprint" chemical species applicable to a wide variety of fields including material science,,${ }^{12,13}$ biochemistry and biosensing, ${ }^{2-6,14-16}$ and electrochemistry. ${ }^{17,18}$ The dominant mechanism for most SERS processes is electromagnetic enhancement from the amplification of light by the excitation of localized surface plasmon resonances.

In conventional SERS sensors, the excitation field is incident normal to the surface and couples to surface plasmon modes associated with flat or randomly roughed precious metal surfaces, which are inherently localized within a few tens of nanometers to the surface of the device. When light is incident on the surface, a plasmon is excited which increases the local field energy in the vicinity of the target molecule thereby enhancing the intensity of the Raman-scattered light.

In this article, we further our investigation into a novel configuration for the excitation of SERS active molecules by fabricating and characterizing nanofeatures etched directly into a slab waveguide core. This provides a first step toward creating a fully integrated multiparameter SERS sensor device, which could be manufactured by low cost, roll-to-roll or sheet level imprinting. In comparison to conventional SERS, the novelty of this article is in the method, in which the SERS measurements are performed. The 785-nm laser probe is coupled in to the slab waveguide core in line with the sensing area. The laser interacts with the sensing region and the vertically projected signal is measured using a Raman probe.

\section{FDTD Simulations}

Finite-difference time-domain (FDTD) method is widely used to numerically simulate photonic devices ${ }^{19}$ and allows calculation of the electromagnetic field as a function of time and space in a given refractive index structure in response to an electromagnetic excitation. A variety of nanofeatures etched into a silicon oxynitride $\mathrm{SiO}_{x} \mathrm{~N}_{y}$ core were modeled ${ }^{20,21}$ using the commercial package FDTD solutions (Lumerical Solutions Inc, Vancouver, British Columbia, Canada). Figure 1 shows the propagating waveguide mode as it interacts with 500-nm diameter, 1000$\mathrm{nm}$ pitch, and 400-nm etched cylindrical nanofeatures. The fine mesh used for the nanostructures was $8 \mathrm{~nm}$ in the $x$ (perpendicular to mode propagation), $y$ (along propagation direction), and

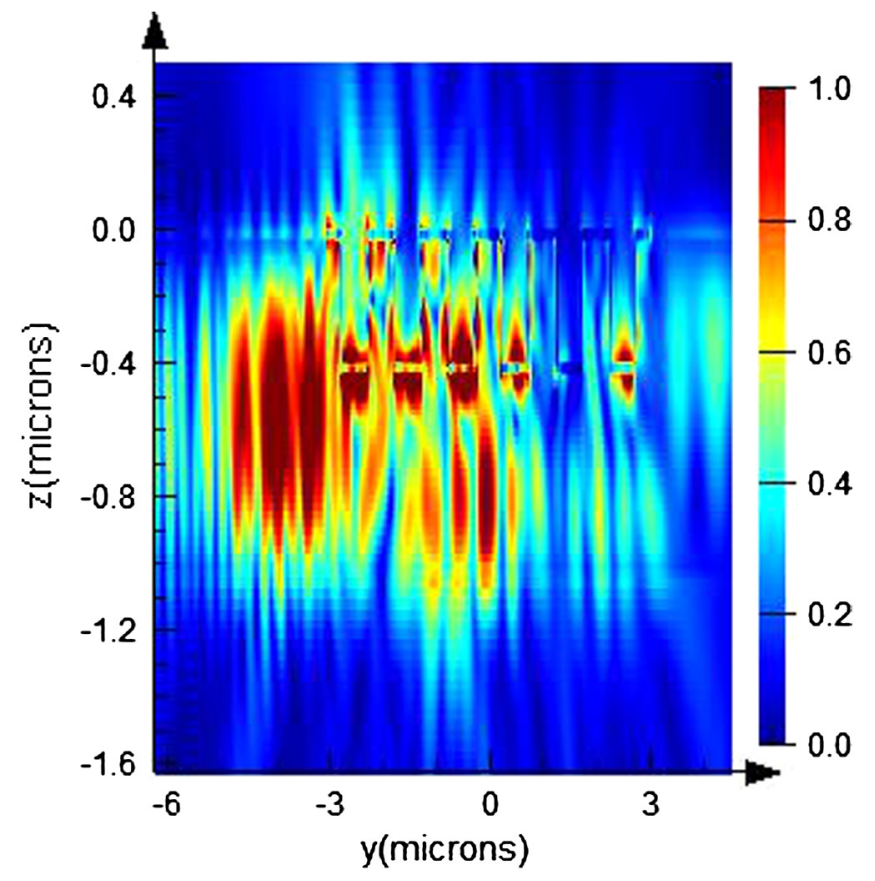

Fig. 1 Lumerical modeling cross section along waveguide/surface enhanced Raman spectroscopy (SERS) sensor for 500-nm diameter, 1000-nm pitch, and 400-nm etched cylindrical nanofeatures. 
$z$ directions with an overall mesh accuracy of 4 . The time step for each simulation was $0.018 \mathrm{fs}$ with the complete simulation time set at $1000 \mathrm{fs}$.

Periodic boundary conditions were used in the $x$ direction and perfectly matched layers (PML) were used in $y$ and $z$ directions. PML boundary conditions absorb the incident light with minimal reflections, by increasing the amount of layers any reflected light can be reduced. For each simulation, the number of PML layers was set at 24. By following standard convergence testing steps, errors in the simulation result were reduced. Initial sweep simulations started with a coarse mesh to reduce any sources of error such as PML reflectivity. The mesh size and accuracy sweep systemically decreased the mesh size around the nanostructures until the result converged. This was achieved using a mesh override region around the area of interest, i.e., the gold-coated nanostructures.

The results show substantial activity within each hole. However, as the mode continues the activity lessens. The results from these FDTD solutions simulations were verified by completing a range of examples of photonic crystal structures given on the Lumerical website. The modeling was then used to test the effect of lattice pitch (1000 to $2500 \mathrm{~nm}, 500 \mathrm{~nm}$ increment), and pit diameter (500 to $2250 \mathrm{~nm}, 250 \mathrm{~nm}$ increment) for a variety of etched nanostructures and was used to determine the optimum design. ${ }^{20,21}$

\section{Device Fabrication}

This section gives details of the prototype-integrated test device layout and fabrication. The aim is to create a silicon-based waveguide-integrated SERS sensor prototype, which could then be mass produced using roll-to-roll or sheet level imprinting techniques. The polymer substrates proposed for the imprinting process will have a refractive index of $\sim 1.7$, therefore for the prototype silicon-based devices, it was necessary to obtain a low loss thin film with similar refractive index. Silicon oxynitride $\left(\mathrm{SiO}_{x} \mathrm{~N}_{y}\right)$ was chosen as a suitable candidate as the refractive index can be tuned between silicon nitride (2.0) and silicon oxide (1.48).

Initial test depositions for the $\mathrm{SiO}_{x} \mathrm{~N}_{y}$ layers were carried out on both square silicon test pieces for measuring the refractive index by ellipsometry, and on thermally oxidized silicon strips for checking waveguide losses and confirming the ellipsometry results using prism coupling. The refractive index at both 800 and $633 \mathrm{~nm}$ [Figs. 2(a) and 2(b)] shows a roughly exponential decay with increasing $\mathrm{N}_{2} \mathrm{O}$ flow rates. There is fair agreement with the results at $633 \mathrm{~nm}$ from both the variable angle ellipsometer and the Metricon (Pennington, New Jersey) prism coupler. The target index of 1.7 was met by using an $\mathrm{N}_{2} \mathrm{O}$ flow rate of $\sim 120 \mathrm{sccm}$. As shown in Fig. 2(c), the TE and TM waveguide losses for the initial correct refractive index layers were $8 \mathrm{~dB} / \mathrm{cm}$.

X-ray photoelectron spectroscopy (XPS) was used on the thin-film layers deposited to determine their stoichiometric ratio. A microfocused monochromated $\mathrm{Al} \mathrm{K} \alpha$ x-ray source was set to illuminate a spot of $400 \mu \mathrm{m}$ in size using $50-\mathrm{eV}$ pass energy and $0.1-\mathrm{eV}$ step size for each element specific scan. During the XPS survey spectrum for each material, the C 1-s peak at a binding energy of $284.8 \mathrm{eV}$ was observed; this was attributed to the surface contamination after exposure to air and was used to verify charge compensation by the low-energy electron flood gun. These results are shown in Fig. 2(d).

To reduce the waveguide losses of the deposited layer, depositions were performed to assess the influence of the plasma frequency on the refractive index and optical losses. A 20-s cycle period was defined whereby the system would deposit in low-frequency (LF, $100 \mathrm{kHz}$ ) mode for a fraction of that period, and in high-frequency (HF, $13.56 \mathrm{MHz})$ mode for the remainder. Increasing the proportion of time spent running in LF mode was found to increase the measured (ellipsometric) refractive index at $\lambda=800 \mathrm{~nm}$ from 1.707 for zero LF power to 1.747 for total LF power. This change in refractive index was compensated for in subsequent runs by increasing the $\mathrm{N}_{2} \mathrm{O}$ flow rate to $210 \mathrm{sccm}$. More importantly, the optical losses measured at $632.8 \mathrm{~nm}$ reduced to $1 \mathrm{~dB} / \mathrm{cm}$, as shown in Fig. 3(b). Using the completely LF process, $1 \mu \mathrm{m}$ lowloss, index-matched $\mathrm{SiO}_{x} \mathrm{~N}_{y}$ was deposited on a $100-\mathrm{mm}$ thermal oxidized silicon wafer for e-beam pattern generation.

In its as-deposited state, $\mathrm{SiO}_{x} \mathrm{~N}_{y}$ deposited by plasma enhanced chemical vapour deposition (PECVD) is heavily contaminated by hydrogen. The percentage of hydrogen incorporated can 


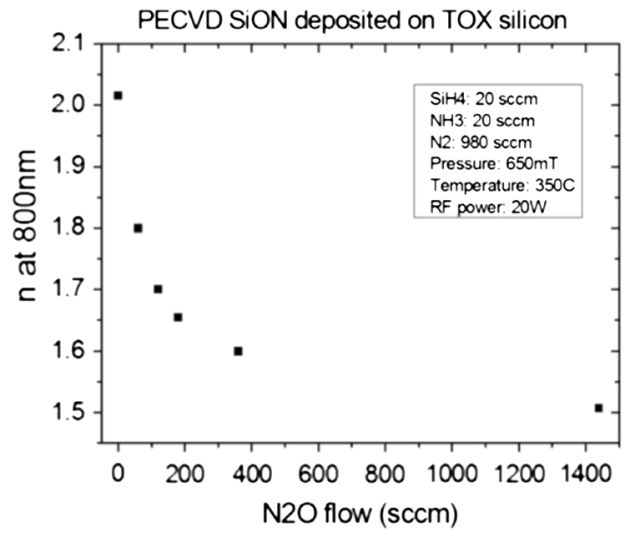

(a)

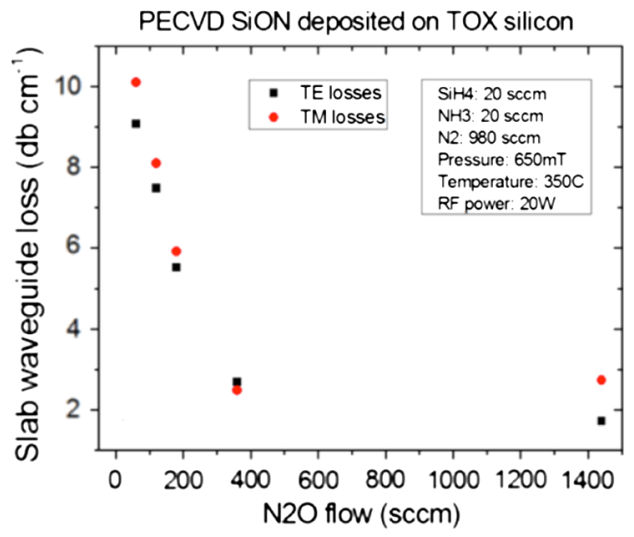

(c)

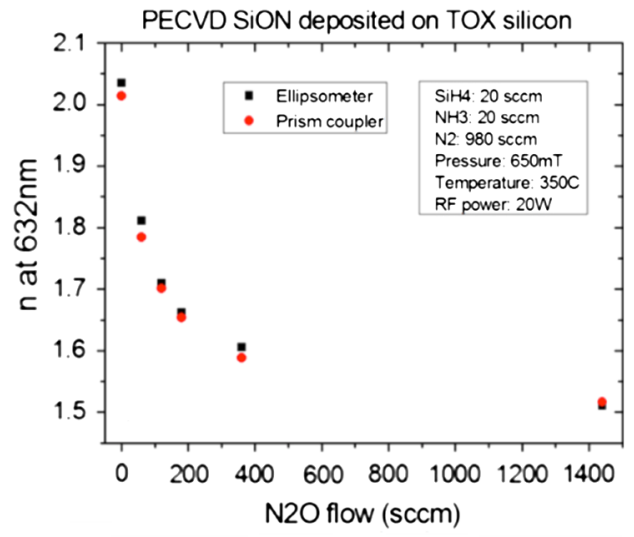

(b)

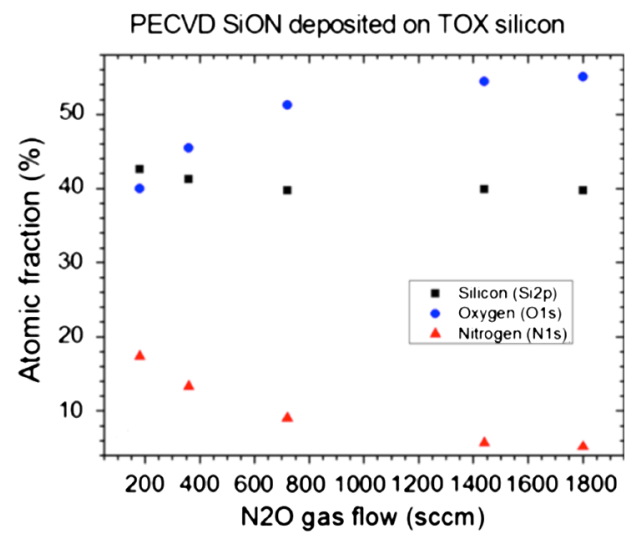

(d)

Fig. 2 Refractive index of plasma enhanced chemical vapor deposition (PECVD) $\mathrm{SiO}_{x} \mathrm{~N}_{y}$ thin films at (a) $\lambda=800 \mathrm{~nm}$ and (b) $\lambda=633 \mathrm{~nm}$ for variable $\mathrm{N}_{2} \mathrm{O}$ flow rates. (c) Corresponding TE/ $\mathrm{TM}$ slab waveguide losses at $633 \mathrm{~nm}$. (d) $\mathrm{SiO}_{x} \mathrm{~N}_{y}$ compositional analysis by x-ray photoelectron spectroscopy.

be up to 20 at. \%, although this fact is not apparent from the measured XPS data owing to the technique's inability to detect such a low-mass atom. The hydrogen contamination, and specifically the $\mathrm{N}-\mathrm{H}$ bonding, is known to cause severe optical losses at telecoms wavelengths whereas losses at visible wavelengths are not seriously affected. A series of LF-deposited samples were annealed for $2 \mathrm{~h}$ at temperatures of $400^{\circ} \mathrm{C}, 600^{\circ} \mathrm{C}, 800^{\circ} \mathrm{C}$, and $1000^{\circ} \mathrm{C}$ in an inert Ar atmosphere. The samples were very slowly $(5 \mathrm{~mm} / \mathrm{min})$ loaded into a tube furnace preheated to $200^{\circ} \mathrm{C}$ and the temperature ramped up to the annealing temperature at $5^{\circ} \mathrm{C} / \mathrm{min}$.

Figure 4 clearly indicates that the already low-loss material was significantly improved to the point, where losses hit the lower sensitivity limit of the measurement system. Although there was little difference in the underlying material absorption at temperatures above $600^{\circ} \mathrm{C}$, there were very noticeable (visible) points of strong scattering for annealing at $800^{\circ} \mathrm{C}$ and above. This was attributed to nucleation of the component materials at these sites. Figure 4 also shows that there is an almost linear decrease in the refractive index as the annealing temperature is increased.

The layout of the device chip is shown in Fig. 5 and was devised to test the effect of lattice pitch (1000 to $2500 \mathrm{~nm}, 500 \mathrm{~nm}$ increment) and pit diameter (500 to $2250 \mathrm{~nm}, 250 \mathrm{~nm}$ increment) in accordance with FDTD simulation results. ${ }^{20}$ Each chip included sensors areas 25, 50, and 100 rows wide in either a 0 or $45-\mathrm{deg}$ orientation. Square and circular straight-sided etched holes were arranged as separate test chips. A 400-nm layer of ZEP520A e-beam resist was spun on top of the wafers and patterned by direct write e-beam lithography.

After development [MIBK:IPA (1:3, $30 \mathrm{~s})$ ], the patterned wafers were etched in a parallel plate reactive ion etch system (OIPT PlasmaLab 80+, Bristol, United Kingdom). The etch was $30 \mathrm{~s}$ in duration using $\mathrm{CHF}_{3} / \mathrm{Ar}(25 / 25 \mathrm{sccm})$ etch chemistry and 200-W RF power. Each etch step was separated by cooling periods of $60 \mathrm{~s}$ using a $\mathrm{N}_{2}(68 \mathrm{sccm})$ gas flow. These two steps 


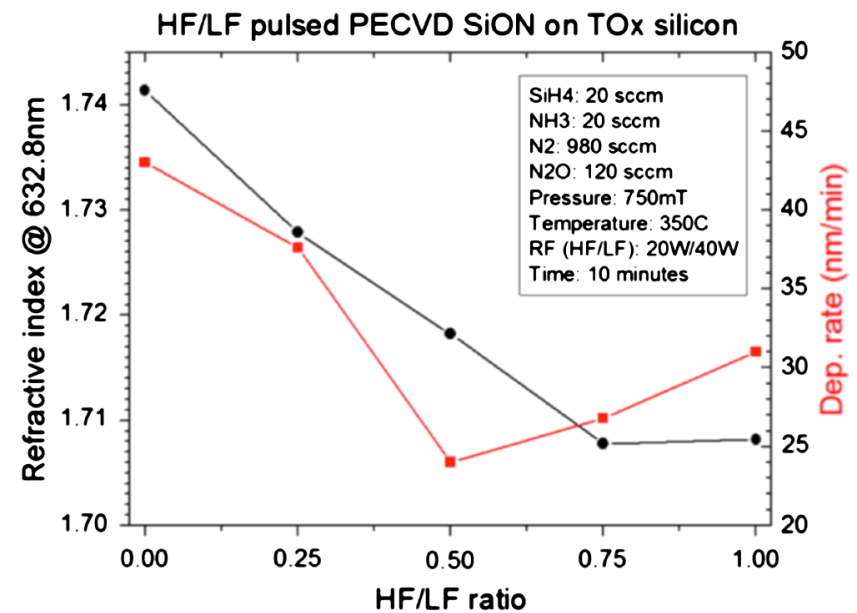

(a)

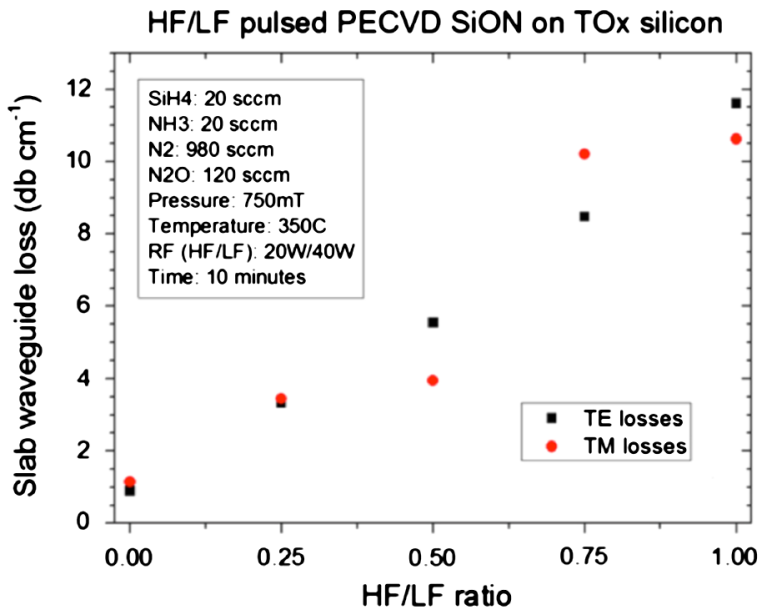

(b)

Fig. 3 Influence of high- and low-plasma frequencies on the refractive index (a) and optical losses (b) of PECVD deposited $\mathrm{SiO}_{x} \mathrm{~N}_{y}$ films.

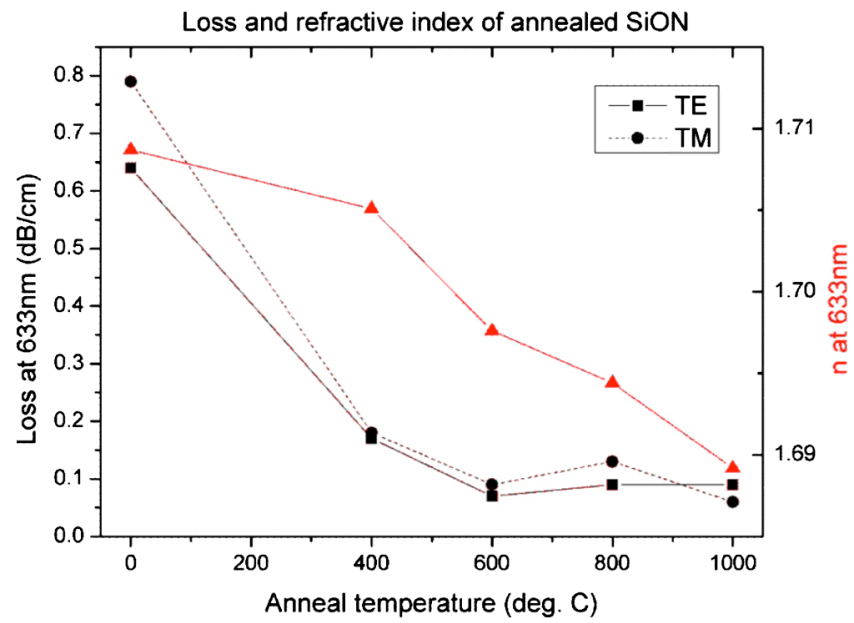

Fig. 4 Optical losses and refractive index of annealed $\mathrm{SiO}_{x} \mathrm{~N}_{y}$ thin films on thermal oxidized silicon at $633 \mathrm{~nm}$. 
were looped 32 times for a total of 16 min etching time. All other process parameters remained constant $\left(18^{\circ} \mathrm{C}, 30 \mathrm{mT}\right)$. The long nitrogen cooling step was found to be necessary to avoid burning the resist mask. Once etched the wafers were then returned to the e-beam tool before metallization to define an open resist window around the nanostructured sensor.

A metal lift off process was used to selectively coat the sensor area with a metal bi-layer consisting of 1-nm $\mathrm{Cr}$ and $25-\mathrm{nm} \mathrm{Au}$ using angled e-gun evaporation process (Leybold Optics LAB700, Alzenau, Germany). The wafer was then left overnight in N-Methyl-2-pyrrolidone (NMP) to lift off the $\mathrm{Cr} / \mathrm{Au}$ around the sensor areas as shown in Fig. 5. This step was done to remove any gold from the input/output waveguide areas thus reducing the possibility of loss due to the interaction of the probe and the Au surface.

\section{Waveguide Coupling}

The fabricated wafer was diced into individual test chips and polished for optical and normal incident Raman measurements. To test coupling from the underlying waveguide to the SERS/ SPR sensor region (metalized holes) a Spectra Physics (Santa Clara, California) Ti:Sapphire laser tuned to $800 \mathrm{~nm}$ was used as a probe source and coupled into the underlying waveguide core using an $f=11$-mm aspheric lens. The nanostructured sensor test chip used was 500-nm diameter etched holes at 1000-nm lattice pitch. Each sensor area was probed and the intensity of light emerging at the output of the waveguide was measured using a Jobin Yvon spectrometer. A small piece of card was placed on top of the test chip to block any residual-uncoupled light, which might otherwise project along the surface of the waveguide. Coupling to the sensor region would in this case be expected to give rise to a reduction in transmission. Figure 6 shows the intensity for each sensor area for (a) 0 deg and (b) 45 deg orientation after two neutral density filters with optical densities 2 ( $1 \%$ transmission) and 3 ( $0.1 \%$ transmission).

By utilizing the waveguide, the sensing region can be interrogated from underneath and any field interaction with the gold nanostructures can be monitored from above the sensor. A Spectra Physics Ti:Sapphire laser tuned to $785 \mathrm{~nm}$ was used as a probe source and coupled into the underlying waveguide core of the 500-nm diameter etched holes at 1000-nm lattice pitch sensor using an $f=11$-mm aspheric lens. A Raman probe (InPhotonics, Norwood, Massachusetts) was positioned over the 25 and 100 row sensor and the laser was aligned so that the waveguide mode was incident on the central area of the etched nanostructures (as shown in Fig. 5). Figure 7 shows the comparison between the 25 and 100 row for the premolecule-coated signal vertically projected from the sensor. As can be seen, regardless of the amount of rows used the light vertically projected and detected by the Raman probe does not change. The peaks seen were attributed to the $\mathrm{SiO}_{x} \mathrm{~N}_{y}$ of the underlying waveguide core.

\section{Conventional Raman Spectroscopy}

Figure 8 shows the normal incident Raman from four different test chips with the same lattice pitch $(1000 \mathrm{~nm})$ but varying hole diameter $(500$ and $750 \mathrm{~nm})$ and etched nanofeatures (circular or square etched holes) after application of benzyl mercaptan $\left(\mathrm{C}_{6} \mathrm{H}_{5} \mathrm{CH}_{2} \mathrm{SH}\right)(\mathrm{BZM})$ Raman active

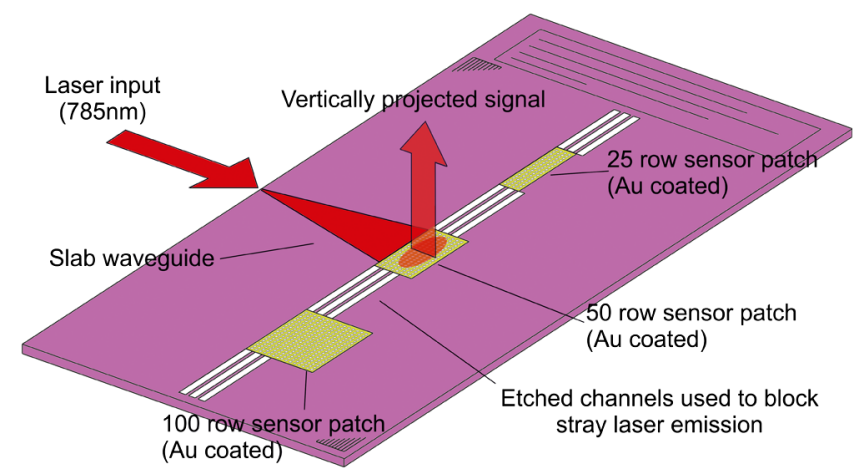

Fig. 5 Nanostructured waveguide core device chip for integrated SERS prototype. 
Pearce et al.: Integrated waveguide and nanostructured sensor platform for surface-enhanced...

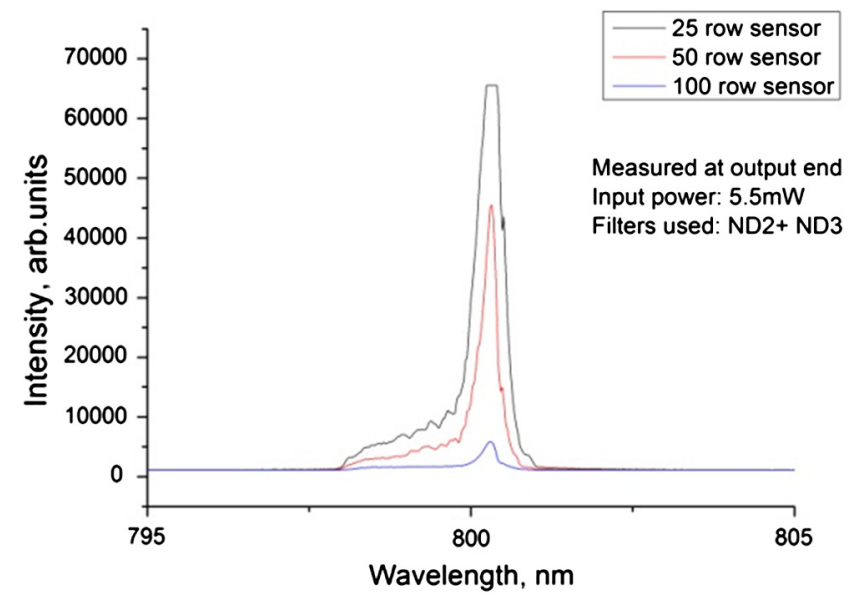

(a)

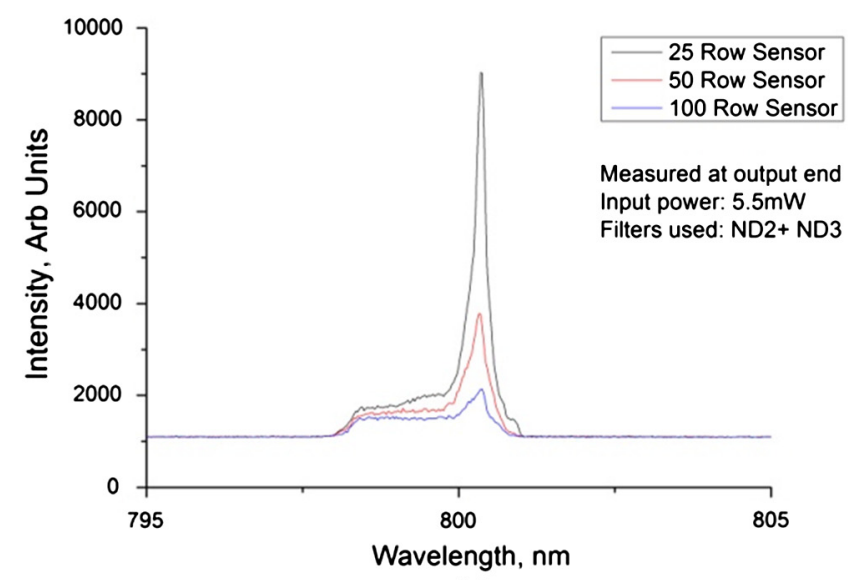

(b)

Fig. 6 Transmission intensity for (a) 0 deg and (b) 45-deg orientation for 500-nm diameter and $1000-\mathrm{nm}$ pitch sensor. Neutral density filters were used to reduce the intensity.

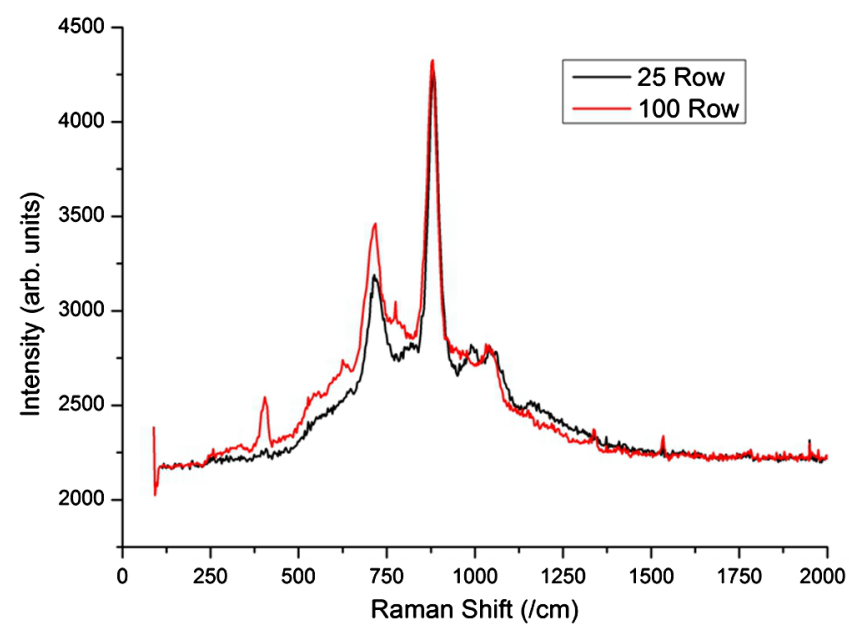

Fig. 7 Vertical projected premolecule-coated Raman probe measurement comparison between 25 and 100 row sensors, 25-nm gold, vertical polarization for 500-nm diameter, 1000-nm pitch circular-etched nanostructures. 
Pearce et al.: Integrated waveguide and nanostructured sensor platform for surface-enhanced...
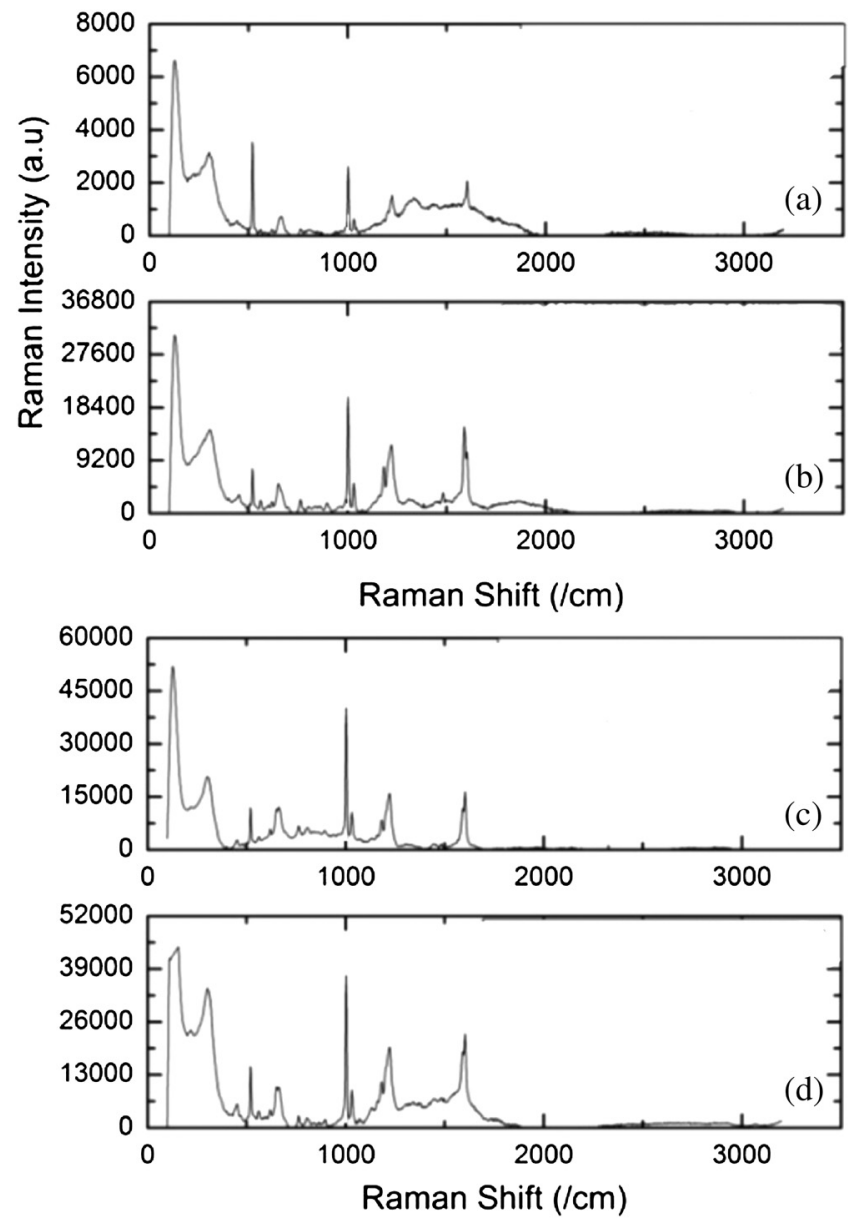

Fig. 8 Raman measurement on BZM-coated test chips for 1000-nm pitch and different diameter etched holes (a) $500 \mathrm{~nm}$ : circular, (b) $500 \mathrm{~nm}$ : square, (c) $750 \mathrm{~nm}$ : circular, and (d) $750 \mathrm{~nm}$ : square. A Raman signal associated with BZM can be seen at $\sim 1000 \mathrm{~cm}^{-1}$ and $\sim 1600 \mathrm{~cm}^{-1}$ for all of the test samples measured.

test molecule. The concentration of the BZM molecule was constant for all test chips at $29.3 \mu \mathrm{L}$ in $50 \mathrm{~mL}$ of ethanol and each test chip was incubated for $1 \mathrm{~h}$ in the mixture.

The results show the devices are SERS active with sharp peaks and relatively high counts (for low thickness of $\mathrm{Au}$ ) noticed at $\sim 1000$ and $\sim 1600 \mathrm{~cm}^{-1}$. These were associated with the trigonal ring "breathing" (990 to $\left.1010 \mathrm{~cm}^{-1}\right)$ and the ring stretches (doublet) $\left(1550\right.$ to $\left.1630 \mathrm{~cm}^{-1}\right)$ of

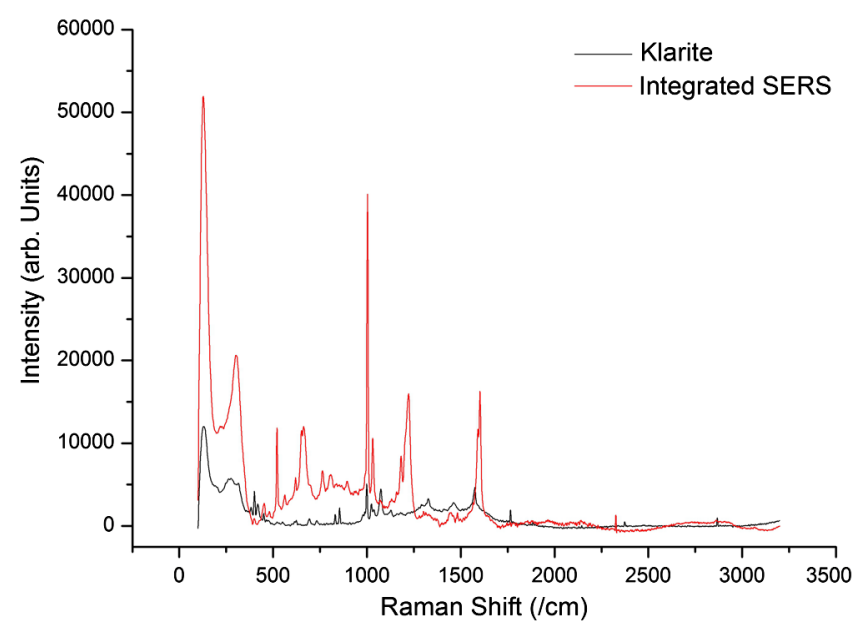

Fig. 9 Raman spectroscopy comparison between Klarite (black) and integrated SERS (red). 
the benzyl mercaptan molecule. In all cases measurement parameters were $20-\mathrm{mW}$ power $(10 \%$ of maximum), 10 -s exposure time, 1 accumulation, 50× magnification and a $0.75 \mathrm{NA}$.

It is interesting to note that $750-\mathrm{nm}$ hole diameter gives larger overall signal level compared with 500-nm diameter, suggesting that designs with high air filling fraction may give better SERS signal. The total number of counts is also relatively high considering the very thin $(25 \mathrm{~nm})$ gold layer. Hence, these measurements prove that the gold thickness and nanostructure morphology is suitable for SERS sensing.

Figure 9 shows the comparison between normal incident Raman measurements for circular etched holes, 1000-nm pitch, 750-nm diameter, silicon oxynitride, and 25-nm Au (red trace) and the standard Klarite ${ }^{\mathrm{TM}}$ test chip (black trace-Pyramidal pits, 2000-nm pitch, 1500-nm diameter, silicon 300-nm Au, Renishaw Diagnostics Ltd., Glasgow, Scotland, United Kingdom). A reduced power $(0.2 \mathrm{~mW})$ was used for the Klarite ${ }^{\mathrm{TM}}$ Raman measurement compared with the integrated test chip $(20 \mathrm{~mW})$. This was attributed to the thickness of the Au used in both cases. The Klarite ${ }^{\mathrm{TM}}$ chip (300-nm Au) is much more reflective than the test chip (25-nm Au) and would therefore give a much higher intensity count at modest power levels due to the reflection of the incident laser power. The fabricated test chips require an optically thin layer of gold to allow for the probe to interact with the coated nanostructures. This result confirms that it is possible to achieve a large intensity signal from a optically thin-coated nanostructured waveguide SERS in a normal incident Raman measurement arrangement.

\section{Conclusions}

Thin films of silicon oxynitride were deposited using PECVD on to thermal oxide coated 4 inch wafers and annealed at various temperatures to obtain low-loss layers suitable for the waveguide core material. The layout of the device wafer was devised to test the effect of lattice pitch (1000 to $2500 \mathrm{~nm}$ ), and pit diameter (500 to $2250 \mathrm{~nm}$ ) in accordance with the simulation results. Each chip included 25, 50, and 100 rows in either 0 deg or 45-deg lattice orientation. Square and circular pits were arranged as separate test chips. This produced approximately 70 different designs to be optically tested. The wafer was diced and polished and optically tested using a Ti:Sapphire laser tuned to $800 \mathrm{~nm}$ with the transmission intensity at the output end measured using a spectrometer. The 25 row sensor gave the highest intensity/transmission as would be expect due to the reduced number of holes. A waveguide Raman experiment was completed using a Raman probe mounted above the sensor area with the Ti:Sapphire laser end fire coupled into the slab waveguide. The measurement was done on a sample without BZM coating. The results showed a strong signal below $1000 \mathrm{~cm}^{-1}$ attributed to the lattice vibrations of $\mathrm{SiO}_{x} \mathrm{~N}_{y}$. Normal incident Raman spectroscopy was performed on several of the BZM-coated samples. The test chips were shown to be SERS active using only $\sim 25$-nm-thick gold. The graphs show sharp peaks and relatively high counts at $\sim 1000$ and $1600 \mathrm{~cm}^{-1}$ associated with trigonal ring "breathing" (990 to $\left.1010 \mathrm{~cm}^{-1}\right)$ and the ring stretches (doublet) $\left(1550\right.$ to $\left.1630 \mathrm{~cm}^{-1}\right)$ of the benzyl mercaptan molecule. It was also shown that designs with high air filling fraction gave better SERS signals. The normal incident Raman results for the integrated SERS prototype were compared with the industry standard Klarite ${ }^{\mathrm{TM}}$ and were shown to have excellent agreement. This result confirms that it is possible to achieve a signal from a thinly coated nanostructured waveguide SERS in a normal incident Raman measurement arrangement.

\section{Acknowledgments}

This work was funded by EU FP7 Project, Photosens- "Capacity building for the development of nanotech-based multiparameter sensors." The main body of work was undertaken by the main author with M. Pollard supplying the PECVD $\mathrm{SiO}_{x} \mathrm{~N}_{y}$ layers, Raman measurements were undertaken by $\mathrm{S}$. Oo, the direct write e-beam lithography was done by R. Chen and S. Kalsi provided the Raman active molecule coating on all test samples. The work was supervised and guided by M. D. B. Charlton.

\section{References}

1. K. Kneipp et al., "Ultrasensitive chemical analysis by Raman spectroscopy," Chem. Rev. 99(10), 2957-2975 (1999), http://dx.doi.org/10.1021/cr980133r. 
Pearce et al.: Integrated waveguide and nanostructured sensor platform for surface-enhanced...

2. W. Xie et al., "Application of surface-enhance Raman scattering in cell analysis," J. Raman Spectrosc. 42(6), 1248-1254 (2011), http://dx.doi.org/10.1002/jrs.2857.

3. Y. S. Huh, A. J. Chung, and D. Erickson, "Surface enhanced Raman spectroscopy and its application to molecular and cellular analysis," Microfluid Nanofluid 6(3), 285-297 (2009), http://dx.doi.org/10.1007/s10404-008-0392-3.

4. T. Vo-Dinh, L. R. Allain, and D. L. Stokes, "Cancer gene detection using surface-enhanced Raman scattering (SERS)," J. Raman Spectrosc. 33(7), 511-516 (2002), http://dx.doi.org/ $10.1002 / \mathrm{jrs} .883$.

5. A. G. Ryder, "Surface enhanced Raman scattering for narcotic detection and applications to chemical biology," Curr. Opin. Chem. Biol. 9(5), 489-493 (2005), http://dx.doi.org/10 .1016/j.cbpa.2005.07.001.

6. R. W. Berg et al., "Ab initio calculations and Raman and SERS spectral analyses of amphetamine species," Appl. Spectrosc. Rev 46(2), 107-131 (2011), http://dx.doi.org/10.1080/ 05704928.2010.520180.

7. D. S. Moore, "Instumentation for trace detection of high explosives," Rev. Sci. Instrum. 75(8), 2499-2512 (2004), http://dx.doi.org/10.1063/1.1771493.

8. H. Wackerbarth et al., "Detection of explosives based on surface-enhanced Raman spectroscopy," Appl. Opt. 49(23), 4362-4366 (2010), http://dx.doi.org/10.1364/AO.49.004362.

9. M. Fleischmann, P. J. Hendra, and A. J. McQuillan, "Raman spectra of pyridine adsorbed at a silver electrode," Chem. Phys. Lett. 26(2), 163-166 (1974), http://dx.doi.org/10.1016/ 0009-2614(74)85388-1.

10. X. Zhang, N. C. Shah, and R. P. Van Duyne, "Sensitive and selective chem/bio sensing based on surface-enhanced Raman spectroscopy (SERS)," Vib. Spectrosc. 42(1), 2-8 (2006), http://dx.doi.org/10.1016/j.vibspec.2006.02.001.

11. M. Muniz-Miranda et al., "SERS investigation on the polymerization of carbazolyl-diacetylene monolayers on gold surfaces," Macromol. Symp. 230(1), 67-70 (2005), http://dx.doi .org/10.1002/masy.200551143.

12. M. G. Blaber, M. D. Arnold, and M. J. Ford, "A review of the optical properties of alloys and intermetallics for plasmonics," J. Phys. Condens. Matter 22(14), 143201 (2010), http://dx .doi.org/10.1088/0953-8984/22/14/143201.

13. M. J. A. Hore, A. L. Frischknecht, and R. J. Composto, "Nanorod assemblies in polymer films and their dispersion-dependent optical properties," ACS Macro Lett. 1(1), 115-121 (2012), http://dx.doi.org/10.1021/mz200031g.

14. R. A. Tripp, R. A. Dluhy, and Y. Zhao, "Novel nanostructures for SERS biosensing," Nanotoday 3(3-4), 31-37 (2008), http://dx.doi.org/10.1016/S1748-0132(08)70042-2.

15. H. T. Ng et al., "Label free DNA biosensor based on SERS molecular sentinel on nanowave chip," Anal. Chem. 85(13), 6378-6383 (2013), http://dx.doi.org/10.1021/ac400763c.

16. K. C. Bantz et al., "Recent progress in SERS biosensing," Phys. Chem. Chem. Phys. 13(24), 11551-11567 (2011). http://dx.doi.org/10.1039/C0CP01841D.

17. Z. Q. Tian, "Surface enhanced Raman spectroscopy: advancements and applications," J. Raman Spectrosc. 36(6-7), 466-470 (2005), http://dx.doi.org/10.1002/jrs.1378.

18. M. A. Tadayyoni, P. Gao, and M. J. Weaver, "Application of surface enhanced Raman spectroscopy to mechanistic electrochemistry: oxidation of iodide at gold electrodes," J. Electroanal. Chem. 198(1), 125-136 (1986), http://dx.doi.org/10.1016/0022-0728 (86)90031-8.

19. H. Rao, R. Scarmozzino, and R. M. Osgood, "An improved ADI-FDTD method and its application to photonic simulations," IEEE Photonics Tech. Lett. 14(4), 477-479 (2002), http://dx.doi.org/10.1109/68.992583.

20. S. J. Pearce et al., "Integration of nanostructures and waveguide core for surface enhance Raman spectroscopy: a novel excitation method," Proc. SPIE 8264, 826412 (2012), http:// dx.doi.org/10.1117/12.910476.

21. S. J. Pearce et al., "FDTD modelling of waveguide core integrated etched nanostructures," Proc. SPIE 8818, 881811 (2013), http://dx.doi.org/10.1117/12.2022207.

Stuart J. Pearce is currently a senior research fellow in the School of Electronics and Computer Science at University of Southampton. His undergraduate degree and $\mathrm{PhD}$ were both completed at the University of Hull, although the latter was part time based predominantly in a small laser 
company manufacturing short pulse diode pumped actively Q-switched solid state lasers and funded entirely by the Royal Commission for the Exhibition of 1851.

Michael E. Pollard is a research fellow at the University of Southampton. His primary research interest is nanophotonics and he is currently researching photonic crystal-based sensors for detection of gas.

SweZin Oo is currently a PhD student at University of Southampton and is completing research on SERS. Her work has involved simulating and optimizing the Klarite ${ }^{\mathrm{TM}}$ test chip on silicon and roll-to-roll polymers.

Ruiqi Chen is currently a research fellow working on direct e-beam lithography. He holds a $\mathrm{PhD}$ in optoelectronics engineering and MSc in microelectronics engineering from the University of Southampton. His undergraduate degree was in electrical and electronic engineering at the University of Bradford.

Sumit Kalsi is currently a PhD student working on the development of a platform for formation of stable lipid bilayers for ion channel electrophysiology. This research uses devices fabricated in a cleanroom using 3-D lithography and photolithography methods.

Martin D. B. Charlton is currently reader in photonics and nanofabrication and holds a prestigious Royal Society Research Fellowship in the School of Electronics and Computer Science at Southampton University. He was awarded the Rank Thesis Prize for his $\mathrm{PhD}$ on photonic crystals in 1999 and has continued research in this field and integrated optics for over 15 years, in particular pioneering work in photonic quasi-crystals, and planar-waveguide-based photonic crystal devices. 